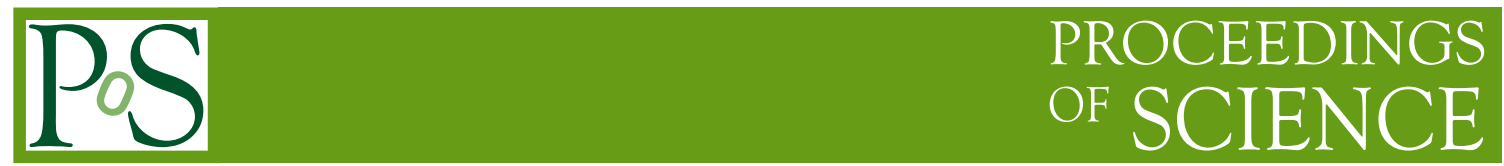

\title{
Introduction of DAQ system for CUP experiments
}

\author{
Jaison Lee* \\ Center for Underground Physics, Institute for Basic Science, Daejeon 34126, Korea \\ E-mail: jsahnleedibs.re.kr
}

\begin{abstract}
CUP, Center for Underground Physics, is one of the research centers belonging to Institute for Basic Science (IBS), Korea. CUP is conducting several experiments in the field of neutrino-less double beta decay, direct WIMP search, and neutrino oscillation, such as COSINE-100, AMoRE, and NEOS experiment. CUP has developed the DAQ system for these experiments including hardware and software. In this proceeding, DAQ system for CUP experiments is introduced focusing on electronics, online DAQ software, and monitoring system.
\end{abstract}

ICHEP2018, International Conference on High Energy Physics

4-11 July 2018

Seoul, Korea

${ }^{*}$ Speaker. 
The Center for Underground Physics (CUP) [1], established as a headquarter research center of Institute for Basic Science (IBS) in 2013 aims to conduct basic researches for beyond standard model physics to deepen our understanding on the origin and structure of the universe. The CUP is mainly conducting three experiments. AMoRE (Advanced Mo-based Rare process Experiment) aims to search for $0 v \beta \beta$ decay of ${ }^{100}$ Mo nuclei using molybdate scintillating crystals operating at milli-Kelvin temperatures. The AMoRE project is a series of experiments. The first phase of the AMoRE, called AMoRE-Pilot [2], has been operating since 2015 with about $1.5 \mathrm{~kg}$ of ${ }^{40} \mathrm{Ca}^{100} \mathrm{MoO}_{4}$ crystals. The ultimate goal of the AMoRE is to achieve a sensitivity that covers the entire $\langle m \beta \beta\rangle$ range that is allowed by the inverted neutrino mass hierarchy. COSINE [3] tries to find the weakly interacting massive particles (WIMP), one of promising candidates for dark matter, with ultra-low radioactivity crystal detectors. The first phase of the COSINE deploying 8 $\mathrm{NaI}(\mathrm{Tl})$ crystals has been receiving data since 2016. NEOS (Neutrino Experiment for Oscillation at Short baseline) [4] is a reactor neutrino experiment at very short baselines to make a precision measurement of the flux and energy spectrum of antineutrinos emitted from nuclear reactors using a Gd-loaded liquid scintillator detector. NEOS is searching for the oscillation signature of sterile neutrinos and test our understanding of the emission of antineutrinos from the fission products in a nuclear reactor.

CUP has developed a DAQ system that can be used commonly in various scintillation-based experiments. This DAQ system (CUP DAQ system) consists of field programmable gate array (FPGA) based trigger system, flash ADC (FADC) and online DAQ and monitoring software, and can be easily adapted to experiment, saving time and effort.

\begin{tabular}{lcccc}
\hline & Resolution (bit) & Sampling Rate (MS/s) & $\begin{array}{l}\text { Number of } \\
\text { analog input }\end{array}$ & $\begin{array}{l}\text { Input } \\
\text { dynamic rage (V) }\end{array}$ \\
\hline FADC500 & 12 & 500 & 4 & 2.5 \\
M64ADC & 12 & 64 & 32 & 2 \\
AMOREADC & 18 & 2 & 16 & 10 \\
\hline
\end{tabular}

Table 1: Tree types of FADC of CUP DAQ system.

The main DAQ electronics, FADC, can store the signal waveform, which enables particle identification using pulse shape discrimination (PSD) that is an essential tool for reducing background in experiments using a scintillation detector. Also, a trigger system utilizing FPGA eliminates the need to use analog frontend electronics. The three types of FADCs are distinguished by sampling rate and resolution, and utilize FPGA for processing the fast digitized signal. The FADCs of CUP DAQ system are summarized at Table 1. The digitized pulse information is stored in the ring buffer and the FPGA reads this buffer and makes a self (channel) trigger through signal processing. With operating self trigger information, a FADC module can decide a local (module) trigger. These triggering logic can be easily modified or added to quickly respond to a variety of experimental configuration. When a decision of trigger is made, digitized pulse information is copied from the ring buffer to DRAM in the FADC. The size of the DRAM is up to 4 Gbytes. This allows one to read and write data without dead time. The raw data in the DRAM is transferred to DAQ computer via USB3 link. When two or more FADC modules are used, the local trigger information generated 


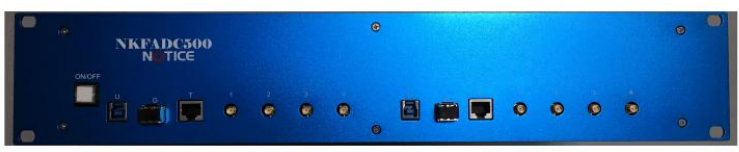

(a)

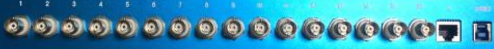

(c)

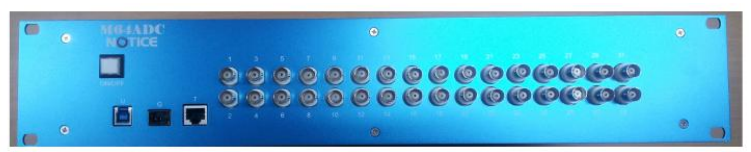

(b)

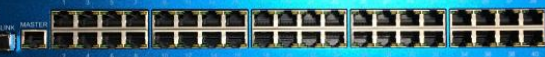

(d)

Figure 1: Electronics of CUP DAQ system, (a) FADC500, (b) M64ADC, (c) AMOREADC, (d) TCB.

by the FADC modules are transmitted to the Trigger Control Board (TCB), and the TCB makes a decision of a global trigger using this information and sends it back to the FADC modules. The TCB also acts as a master clock that synchronizes the time of the FADC modules. With the fastest sampling rate, FADC500 is suitable for scintillation counters with fast decay time or low energy threshold detector at a single photoelectron level. COSINE-100 and NEOS have been adopting FADC500 as their main ADC. AMOREADC is a FADC only for the AMoRE experiment and has a slow sampling rate and a wider dynamic range to handle very slow signals coming from a lowtemperature detector. SADC is the same in all respects to other ADCs, but stores the pulse area without storing the waveform. All experiments in the CUP use the SADC for muon veto detector where a waveform is not critical. Figure 1 shows the front view of the electronics. The DAQ electronics and FPGA logic have been developed and maintained in cooperation with NOTICE CO. Ltd. [5]

Online DAQ software package is a collection of software that provides run configuration and control, data readout, reformatting and writing data and the monitoring system. All software are developed in C++ with the help of ROOT. The Data Logger is a single server and receives triggered data from the Data Receiver to format into an Event. The events created by the Data Logger are written to disk. The Data Logger also distributes events to client applications such as Online Monitoring and Online Event Display through TCP/IP. The Run Control system provides overall control of the DAQ system. All DAQ components have a predefined state model and, under the command of Run Control, make transitions between states in that state model. Run Control is developed using a client/server framework and is written in Python using Qt tools to implement the Graphical User Interface (Figure 2(a)). The monitoring system is used to monitor the health and

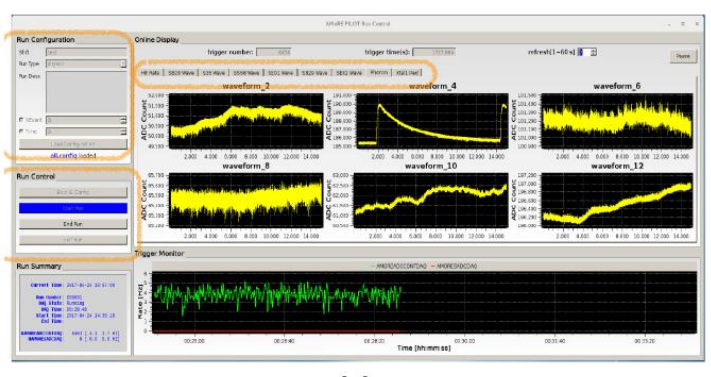

(a)

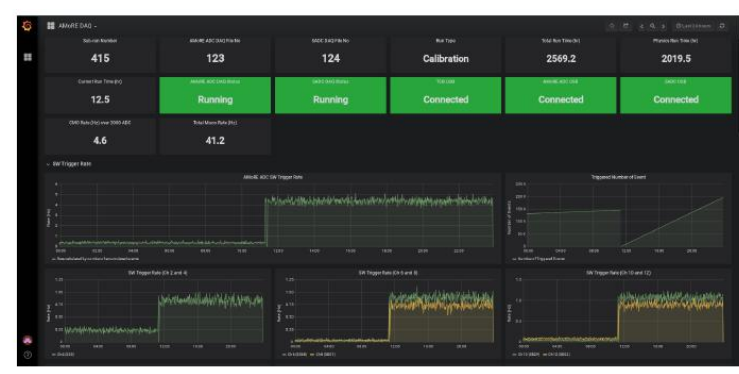

(b)

Figure 2: Online DAQ Utilities, (a) Run control GUI, (b) Web based Run Monitoring 


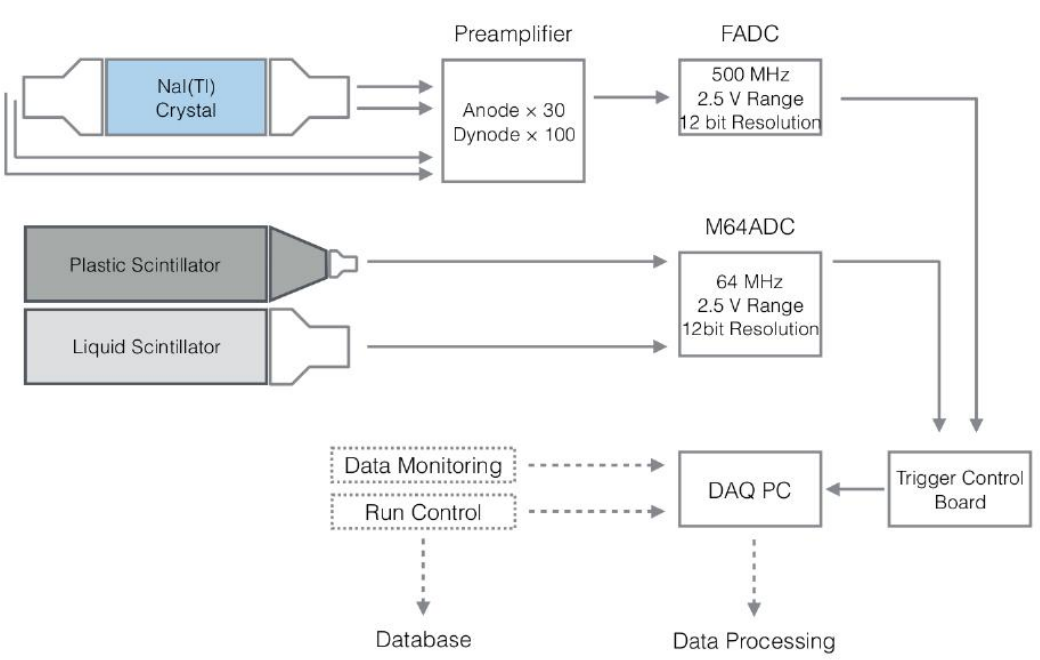

(a)

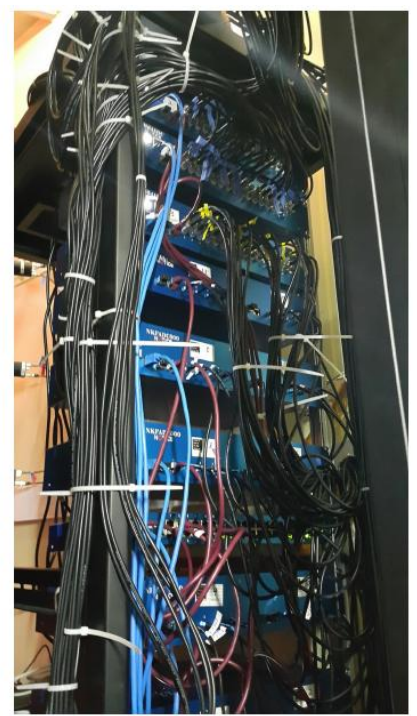

(b)

Figure 3: COSINE-100 DAQ System, (a) Schematic diagram of DAQ system, (b) Photo of installed DAQ hardware

performance of the DAQ system. Using Grafana and InfluxDB, the monitoring system enables us to monitor various parameters on the web in real time (Figure 2(b)).

The CUP DAQ system has been developed with an emphasis on applicability for use in scintillator-based experiments as well as for HPGe detectors and even for small experiments done in the lab, and successfully used in many experiments of the CUP. Figure 3 shows an application to COSINE-100 experiment of the CUP DAQ system. By using a digital pulse processing technique using FPGA, the DAQ electronics can save time and effort when applied to various experiments.

\section{References}

[1] https://cupweb.ibs.re.kr

[2] G.B. Kim et al., Astropart. Phys. 91, 105-112 (2017)

[3] G. Adhikari et al., JINST 13, no. 09, P09006 (2018)

[4] Y.J. Ko et al., Phys. Rev. Lett. 118, 121802 (2017)

[5] https://www.noticekorea.co.kr 